\title{
Review
}

\section{Recent Progress in Diagnosis Methods for Latent Tuberculosis Infection and Its Clinical Applications}

\author{
Ling Zhou
}

Tianjin Lung Cancer Institute, Tianjin Medical University General Hospital, Tianjin China

\section{Keywords \\ Mycobacterium tuberculosis; Latent infection; Tuberculin skin test; interferon-gamma release assays}

\section{Correspondence}

Ling Zhou,

E-mail: lingzhoutjmu@163.com

DOI: $10.1515 / \mathrm{ii}-2017-0110$

\begin{abstract}
Most people with latent Mycobacterium tuberculosis infection can partly develop active tuberculosis (TB). Therefore, diagnosis of this condition bears significance in early TB prevention. To date, the main methods for diagnosis of latent TB infection (LTBI) include tuberculin skin test and interferon $\gamma$ release test. These two methods feature their own advantages and disadvantages. Although new diagnostic markers continually emerge, no uniform diagnostic criteria are available for TB detection. This study summarizes several methods for diagnosis of LTBI and new related markers and their application value in clinical practice.
\end{abstract}

Mycobacterium tuberculosis (MTB) causes the chronic infectious disease tuberculosis (TB), which seriously endangers people's health. The number of TB cases increased worldwide since mid-1980s. Approximately 2 billion people worldwide are infected with MTB, and this condition leads to approximately 8 million new cases and 3 million deaths each year ${ }^{[1]}$. The United Nations classified $\mathrm{TB}$ as one of the three major diseases of the 21st century. In China, the number of TB patients approximates 1,300,000, ranking second in the world. China became one of 22 countries in the world with severe TB cases. Therefore, TB is one of the critical diseases which the Chinese government wants to control. Some studies showed that $5 \%-10 \%$ of latent TB infection (LTBI) population can transform into active $\mathrm{TB}^{[2]}$. LTBI is a subclinical state, which indicates that a patient is infected with $\mathrm{TB}$, but sputum smear result is negative with no radiological sign, and bacteria exist in resting state. Therefore, early $\mathrm{TB}$ diagnosis poses a challenge. LTBI also affects a large number of people. Thus, rapid and specific method for diagnosing LTBI bears significance in TB control.

\section{Tuberculin skin test (TST)}

TST is the basic method for detecting latent infection of
MTB. Purified protein derivative (PPD) is subcutaneously injected into $1 / 3$ of the upper arm by using Mantoux method. All organisms infected with MTB produce corresponding sensitized lymphocytes with recognition ability of the bacterium. When confronted with a small number of MTB or tuberculin, sensitized T lymphocytes stimulated by the same antigen release different soluble lymphatic factors. This phenomenon causes increase in vascular permeability, local aggregation of macrophages, and infiltration. Within approximately $48-72 \mathrm{~h}$, red and hard, and other positive reactions are observed in local areas. When the subject is not infected with MTB, no allergic reaction occurs in locally injected areas. Positive TB is common among those with currently positive MTB infection, past MTB infection, and bacillus CalmetteGuérin (BCG) vaccination. For people with strong positive reaction, lasting reaction occurs without fading. In young populations, probability of newly MTB infection is high. The first PPD test shows negative or weakly positive results. After one week, the second test shows positive result, with an increase of more than $6 \mathrm{~mm}$ in injected areas. This phenomenon is called enhancement effect and also suggests new infection. As a diagnostic method for LTBI, use of TST lasted for nearly a century. However, this method presents many shortcomings. In our country, 
with high vaccination rates and high incidence of $\mathrm{TB}$, TST skin tests result in high false positive rates ${ }^{[3]}$. For people with BCG, nontuberculous mycobacteria (NTM) infection, and nonsensitized individuals, blood input of sensitized individuals causes false positive result. TST features low specificity. For persons with low immunity or immunosuppression, sensitivity of this method is also low [4].

PPD is a multiprotein complex. This protein mainly undergoes old tuberculin, PPD, PPD-S, PPD-S2, and PPD RT23 stages ${ }^{[5]}$. However, several studies reported composition of PPD. PPD also became a bottleneck in studies of molecular mechanism of delayed-type hypersensitivity (DTH), thus inspiring further research. In recent years, research on $P P D$ focused on use of single proteins in PPD to diagnose TB infection. Single proteins exhibit high specificity, but their sensitivity is not ideal. This condition poses difficulty in developing candidate proteins [such as early secretory antigenic target-6 (ESAT-6), culture filtrate protein-10 (CFP-10), MPT64, diphenylphenylediamine, and Rv0934] into second generation of reagents for diagnosis of TB infection. Cocktail protein combination or one protein combined with a variety of protein antigens may solve this problem. Yang et al. ${ }^{[6]}$ pointed out in their study that PPD comprises hundreds of proteins. Among these proteins, DnaK and GroEL2 present the highest amounts. Then, with proteomics methodology, six proteins, namely, Rv0009, Rv0475, Rv0569, Rv0685, Rv2626c, and Rv2632c, were determined to be related to these two proteins. Subsequently, the same researchers compared these six proteins with DTH induced by PPD in terms of strength. Initial results showed absence of difference between strength of DTH reaction induced by DnaK/ GroEL2/Rv0009 and DnaK/GroEL2/Rv0685 and that which was induced by PPD. Further experiments showed that DTH reaction induced by DnaK/GroEL2/Rv0009 and DnaK/GroEL2/Rv0685 is mediated by the activated $\mathrm{CD} 4+/ \mathrm{CD} 8+\mathrm{T}$ cell interacting with interferon (IFN) $-\gamma$ and tumor necrosis factor (TNF)- $\alpha$ rather than INF-10. Other combinations presented poorer results in comparison with PPD. These findings may be caused by interaction between proteins. This protein combination can be used to realize mechanism of DTH and also provide a platform for development of second generation of rapid and sensitive diagnostic reagents for TB infection.

\section{IFN-Y release assay (IGRA)}

IGRA is a new method for detecting MTB infection. This method selects ESAT- 6 and CFP-10 coded in generally missing one region of difference (RD1) in BCG and a large number of nontuberculous mycobacteria (excluding Mycobacterium kansasii, Mycobacterium marinum, and Sujoy Mycobacterium) as specific stimulant. Enzymelinked immunospot assay (ELISPOT) or enzyme-linked immunosorbent assay (ELISA) is used to monitor in vitro activated immune cells or immune molecular weight to diagnose occurrence of TB infection without cross immunity with BCG vaccine and mycobacterium in environment and absence of influence by immune environment of the $\operatorname{organism}^{[5,6]}$.

In 1996, Mahairas et al. discovered RDl and identified it in MTB ${ }^{[5]}$. ESAT-6 and CFP-10 are positioned in Rv3874 and Rv3875 in RD1 region of MTB genome, respectively [7]. These two kinds of protein exhibit high strain specificity and are absent in BCG and in most NTMs, including Mycobacterium avium-intracellulare. According to reports of foreign countries, sensitivity of RDl to MTB infection reaches $85 \%-90 \%$, and specificity is $91 \%-96 \%{ }^{[8]}$. Brock et al. ${ }^{[9]}$ used recombined ESAT- 6 and CFP-10 to perform IGRA for BCG inoculator, nonBCG inoculator, and TB patients, and results were compared with those for PPD. Results showed that IGRA with PPD as stimulating antigen cannot effectively distinguish BCG inoculator and TB patients. Recombined ESAT- 6 and CFP-10 can specifically distinguish MTB-infected person, BCG inoculator, and nonpathogenic mycobacteria infection.

To date, clinical detection method based on IGRA principle was updated four times. QuantiFERON-TB test (QFT), QFT Gold test (QFT-G), and QFT Gold In-Tube (QFT-GIT) test correspond to the first, second, and third generations of systems of this series, respectively. By using ELISA, differences in IFN- $\gamma$ between sera stimulated and not stimulated by related antigen is used as criterion for clinical diagnosis of TB. SPOT ${ }^{\circledR}$ TB test (T-SPOT) is the fourth generation of diagnosis system of TB based on IGRA. To assess test results, ELISPOT is used to detect the number of peripheral blood mononuclear cells releasing INF- $\gamma$.

Compared with PPD test, advantages of IGRA are as follows: (1) high specificity is not affected by BCG and most NTM; (2) objective interpretation; (3) experiment in vitro 
for 24-48 h can acquire results, follow-up is not needed, and missing rate is decreased; and (4) higher sensitivity to immunocompromised and immunosuppressed populations than PPD. However, some limitations are also observed: (1) owing to in vitro tests, laboratory requirements must be fulfilled. Collected samples are immediately treated for tests to avoid decline of cell function. (2) High cost is the main obstacle to popularity in less developed areas. (3) This method cannot predict progress of TB. Lastly, (4) adequate clinical data are lacking. We cannot evaluate children under five years old, individuals exposed to MTB, immunosuppression, and suspicious patients who repeatedly used IGRA tests.

\section{TST and IGRA for evaluating LTBI diagnosis in different populations}

\section{Simple LTBI suspicious person}

Some countries published or updated IGRA operating guidelines. However, some differences were observed in considered conditions. Previously, TST displayed high sensitivity, which is suitable for past or long-term infection of LTBI. IGRA is suitable for current or newly infected LTBI. LTBI is hidden; thus, theoretically, joint detection is a reliable strategy. However, this method also depends on economic situations and local medical conditions. A metaanalysis showed good specificity of IGRA. QFT-GIT is 99\% in unvaccinated BCG population, $96 \%$ in vaccinated BCG population, and in 93\% T-SPOT. TST presents high specificity of $97 \%$ in unvaccinated BCG population, but it is not ideal in vaccinated BCG population. TST and IGRA exhibit inconsistent sensitivities. T-SPOT (90\%) shows higher sensitivity than QFT-GIT (70\%) and TST (77\%) ${ }^{[10]}$. In case of inconsistent results, TST+/IGRAs- is mainly caused by BCG vaccination and NTM infection. TST/IGRAs+ is common in elderly. Possibly, when considering this population, $T$ cell-related immunity significantly decreases in TST than in IGRA ${ }^{[11]}$.

\section{HIV infected person}

Both TST and IGRA are immune-mediated and indirect diagnostic methods. For immunosuppressive patients, sensitivity of these detection methods inevitably decreases, particularly for commonly observed HIV/TB coinfected individuals. Reportedly, incidences of LTBI in HIV-infected population, including AIDS patients receiving antiviral treatment, are significantly higher than that in the normal population ${ }^{[12]}$. Approximately 1/3 of HIV-infected persons also experience TB infection ${ }^{[13]}$. Based on diagnosis of LTBI in HIV-infected population by using TB antigen specificity of INF- $\gamma$ ELISPOT detection technology, Hui et al. ${ }^{[14]}$ reported significantly higher positive rate of ELISPOT in populations with HIV infection and HIV/AIDS and who are receiving antiviral therapy than in healthy people with negative HIV. HIV-infected population yields significantly lower positive rate of PPD than ELISPOT detection. This method is recommended for adjuvant diagnosis of LTBI in AIDS. Some studies showed significantly lower positive rate of PPD in HIV/AIDS patients than normal population. Particularly in AIDS patients with CD4 cell count less than 50 cells $/ \mu \mathrm{L}, \mathrm{PPD}$ result is usually negative or without reaction ${ }^{[15]}$. These findings indicate correlation of CD4 cell count with PPD sensitivity. Therefore, even when PPD is negative, LTBI cannot be completely excluded clinically. Combination of TST with IGRA aids in screening HIVinfected individuals with LTBI. Both sensitivities of these methods to the above populations are significantly lower than those without infection. Degree of decline is related to progress of HIV/AIDS. However, in some cases, T-SPOT performs better than QFT and TST ${ }^{[16]}$.

\section{Patients with autoimmune diseases}

For inflammatory bowel disease, rheumatic diseases, ankylosing spondylitis, and other autoimmune diseases, TNF- $\alpha$ in proinflammatory cell factors in vitro play an important role in disease progress. Serious cases use immunomodulatory drugs (glucocorticoid) or biological agents (etanercept, infliximab, adalimumab, and TNF- $\alpha$ blocking agents). However, TNF- $\alpha$ is also an important component of host against pathogens. Such anti-TNF- $\alpha$ treatment leads to decreased immunity, thus increasing risk of opportunistic infections in patients. Evidence showed that reoccurrence of LTBI is related to application of TNF- $\alpha$ monoclonal antibody. TB infection risk of patients with rheumatoid arthritis and using infliximab and adalimumab is three to four times higher than that using etanercept ${ }^{[17]}$. To date, in comparison with TST, some reports showed higher specificity and sensitivity of IGRA in screening of LTBI patients with inflammatory bowel disease and who did not use TNF- $\alpha$ blocking agent. Thus, IGRA can be used to replace TST ${ }^{[18]}$. Starting from guaranteed sensitivity, TST combined with IGRA proves to be feasible in detecting 
suspicious LTBI with immunosuppression.

\section{Patients with chronic renal failure}

Chronic renal failure population, particularly patients with uremia, also represents a LTBI high-risk group because of their immune function and nutritional disorders. Hemodialysis can be complicated by a variety of infections and other factors. Most TB diagnostic guidelines that were published in many countries recommend two steps for the general population. However, all guidelines suggest that IGRAs should be used directly in immunosuppression to avoid possible false negative in TST ${ }^{[19]}$. Despite combined detection, probability of inconsistency is more significant in China when using BCG routine vaccination. A prospective multicenter clinical trial showed significantly higher probability of TB infection in patients receiving hemodialysis after less than 12 months than those receiving long-term hemodialysis, and this finding suggests that one-time TST per year must be changed to routine screening as basis for admittance ${ }^{[20]}$. Another cohort study approved of generally increasing TB infection risk in hemodialysis patients. However, in absence of other risk factors, absolute risk of TB infection is low. Therefore, LTBI is not necessarily screened when only risk factors are considered (particularly in TB endemic countries) ${ }^{[21]}$.

Two cost performance analyses on IGRA suggested that IGRA, with higher cost performance than TST in high-risk population and close contacts, can compromise for its high price through reducing costs of chest X-ray and medicine prevention. TST/IGRA combined detection is more cost effective than IGRA or TST alone. Among these treatments, cost performance of TST is the lowest ${ }^{[22,23]}$.

\section{Potential new markers of TB}

\section{Interleukin-2 (IL-2)}

In addition to IFN- $\gamma$ and TNF- $\alpha$, IL-2 also plays an important role in human immune process. IL-2, one of the cytokines produced by activated $\mathrm{T}$ cells, can enhance killing ability of natural killer cells, promote B cell proliferation and antibody secretion, and activate macrophages to strengthen immunity. According to different cytokines that they secrete, $\mathrm{T}$ cells are divided into three types, which are as follows: central memory $\mathrm{T}$ cells that generally produce IL-2; effect-memory $T$ cells that mainly secrete IL-2 and INF- $\gamma$; and effect $T$ cells that mainly secrete INF- $\gamma{ }^{[24]}$.
Central memory $\mathrm{T}$ cells display long survival time with IL-2 as their main secretion. These findings represent longterm LTBI state or healed TB. IFN- $\gamma$ corresponds to new MTB infection. IL-2 ELISPOT method can stimulate the immune system to produce MTB-specific T cell through RD1 region antigen, causing the latter to generate IL-2, which can be quantitatively detected by ELISPOT. Some clinical experiments showed that in screening of suspicious objects, combined application of IGRA and IL-2 ELISPOT can improve accuracy of new TB infection. For patients with changing low-to-high IL-2/INF- $\gamma$ ratio, this method indicates outcome of TB infection. Therefore, preventive treatment is not needed ${ }^{[25]}$.

\section{INF- $\gamma$ induced protein 10 (IP-10) and monocyte chemoattractant protein-2 (MCP-2)}

After infection by various pathogenic bacteria, by secreting INF- $\gamma$, T cells and other inflammatory mediators stimulate innate cells and infiltrate cells in inflammatory tissues to produce IP-10 and MCP-2. From the above pathway, highest values of IP-10 and MCP-2 are based on significant amplification effects, which can improve sensitivity of IGRA in screening of LTBI, particularly for high-risk populations with low immunity or suppression. The present literature on diagnosis of LTBI with IP-10/MCP-2 drew positive conclusions. Thus, such findings may serve as important markers ${ }^{[26,27]}$.

\section{Conclusions}

LTBI occurs in huge populations. To date, no golden standard is available for its early diagnosis. In patients with simple LTBI or with underlying disease, specificity and sensitivity of IGRA are significantly higher than those of PPD. However, in some complex cases, accurate diagnosis cannot be attested. In developing countries, major obstacles to popularity of IGRA include its high costs and high requirements for laboratory testing. Guidelines of various countries differ for applicable populations. Combined detection is appropriate for TB diagnosis. Markers in the present clinical detection methods and studies comprise immune mechanism of $\mathrm{T}$ cell response to $\mathrm{RD} 1$ region. Several studies focused on other specific gene regions. New markers are available but still lacking sufficient clinical evidence. For developing countries, such as China, with high TB burden, future research should continuously explore development of cheap detection methods that are 
easy to operate, particularly in detection of LTBI with low immunity.

\section{Declarations}

No.

\section{Competing interests}

The author declares that she has no competing interest.

\section{Authors' contributions}

L Zhou made the literature analysis and wrote, discussed and revised the manuscript of this review.

\section{References}

1 Dye C, Scheele S, Dolin P, et al. Consensus statement. Global burden of tuberculosis: estimated incidence prevalence, and mortality by country. JAMA, 1999, 282(7): 677-686.

2 Shen $\mathrm{HB}$, Wang HH. Reactions advances in therapeutic anti-tuberculosis vaccines. J Microbes Infect, 2010, 5(2):111-116.

3 Chen XX, Yang QT, Xu LM, et al. Diagnosis of latent Mycobacterium tuberculosis infection in undergraduate student of Shenzhen using ELISPOT and PPD skin test. J Clin Pulmonary Med, 2009, 14(6):737-738.

4 Alvarez-Leon EE, Espinosa-Vega E, Santana-Rodriguez E, et al. Screening for tuberculosis infection in Spanish healthcare workers: Comparison of the QuantiFERON-TB gold in-tube test with the tuberculin skin test. Infect Control Hosp Epidemiol, 2009, 30(9):876-883.

5 Yang HL, Kruh-Garcia NA, Dobos KM. Purified protein derivatives of tuberculin--past,present, and future. FEMS Immunol Med Microbiol, 2012, 66(3):273-280.

6 Yang HL, Troudt J, Grover A, et al. Three protein cocktails mediate delayed-type hypersensitivity responses indistinguishable from that elicited by purified protein derivative in the guinea pig model of Mycobacterium tuberculosis infection. Infect Immun, 2011, 79(2):716-723.

7 Skinner MA, Ramsay AJ, Buchan GS, et al. A DNA prime-live vaccine boost strategy in mice can augment INF- $\gamma$ responses to mycobacterial antigens but dose not increase the protective efficacy of two attenuated strains of Mycobacterium bovis against bovine tuberculosis. Immunology, 2003, 108(4):548-555.

8 Codecasa L, Mantegani P, Galli L, et al. An in-house RD1-based enzymelinked immunospot-gamma interferon assay instead of the tuberculin skin test for diagnosis of latent Mycobacterium tuberculosis infection.J Clin Microbiol, 2006, 44(6):1944-1950.
9 Peng Z, Zhu CM. Research progress of Mycobacterium tuberculosis RD1.Chongqing Med, 2011, 40(1):89-98.

10 Hougardy JM, Schepers K, Place S, et al. Heparin-bindinghemagglutinin-induced INF-gamma release as a diagnostic tool for latent tuberculosis. PLoS One, 2007, 2(10):e926.

11 Brock I, Munk ME, Kok-Jensen A, et al. Performance of whole blood IFN- $\gamma$ test for tuberculosis diagnosis based on PPD or the specific antigens ESAT-6and CFP-10.Int J Tuberc Lung Dis, 2001, 5(5):462-467.

12 Pai M, Zwerling A, Menzies D, et al. Systematic review:Tcell-based assays for the diagnosis of latent tuberculosis infection: an update. Ann Intern Med, 2008, 149(3):177-184.

13 Nienhaus A, Schablon A, Diel R. Interferon-gamma release assay for the diagnosis of latent TB infection-analysis of discordant results, when compared to the tuberculin skin test.PloS One,2008,3(7):e2665. DOI:10.1371/journal.pone.0002665

14 Liu FR, Ye LX. The progress in diagnosis of latent tuberculosis infection in HIV-infected populations. J Pub Health Prev Med, 2006,17(5):49-51.

15 Corbett EL, Watt CJ, Walker N, et al. The growing burden of tuberculosis: global trends and interactions with the HIV epidemic.Arch Intern Med, 2003, 163(9):1009-1021.

16 Wang H, Tan Y, Zhu XY, et al. Use of INF- $\gamma$ release assay in latent tuberculosis infection diagnosis in general and HIV-infected populations. Chin J Microb Immunol, 2009, 29(11): 1037-1041.

17 Zeng XA. Primary analysis of HIV infection in patients with pulmonary tuberculosis. Chin J AIDS/STD, 2004, 10(5):354-355.

18 Talati NJ, Gonzalezn-Diaz E, Mutemba C, et al. Diagnosis of latent tuberculosis infection among HIV discordant partners using interferon gamma release assay.BMC Infect Dis, 2011,11:264.

19 Dixon WG, Hyrich KL, Watson KD, et al. Drug-specific risk of tuberculosis in patients with rheumatoid arthritis treated with anti-TNF therapy: results from the British Society for Rheumatology Biologics Register (BSRBR). Ann Rheum Dis, 2010, 69(3):522-528.

20 Sellam J, Hamdi H, Roy C, et al. Comparison of in vitro-specific blood tests with tuberculin skin test for diagnosis of latent tuberculosis before anti-TNF therapy. Ann Rheum Dis, 2007, 66(12):1610-1615.

21 Segall L, Covic A. Diagnosis of tuberculosis in dialysis patients: current strategy. Clin J Am Soc Nephrol, 2010, 5(6):1114-1122.

22 Sayarlioglu H, Gül M, Eren Dagli C, et al. QuantiFERON-TB Gold test for screening latent tuberculosis infection in hemodialysis patients. Tuberk Toraks, 2011, 59(2):105-110.

23 Christopoulos AI, Diamantopoulos AA, Dimopoulos PA, et al. Risk factors for tuberculosis in dialysis patients: aprospective muti-center clinical trial. BMC Nephrol,2009,10:36.

24 Dobler CC, McDonald SP, Marks GB. Risk of tuberculosis in dialysis patients: a nationwide cohort study. PLoS One, 201, 6(12):e29563.

25 Nienhaus A, Schablon A, Costa JT, et al. Systematic review of cost and cost-effectiveness of different TB-screening strategies. BMC Health Serv 
Res, 2011, 11:247.

26

oran A, Booth H, Miller RF, et al. Different screening strategies (single or dual) for the diagnosis of suspected latent tuberculosis: a cost effectiveness analysis. BMC Pulm Med, 2010,10:7. [27] Day CL, Abrahams DA, Lerumo L, et al. Functional capacity of Mycobacterium tuberculosis-specific $\mathrm{T}$ cell responses in human is associated with mycobacterial load.J Immunol, 2011,187(5):2222-2232.
28 Ruhwald M, Bodmer T, Maier C, et al. Evaluating the potential of IP10and MCP-2as biomarkers for the diagnosis of tuberculosis.Eur Respir J, 2008, 32(6):1607-1615.

29 Kabeer BS, Raja A, Raman B, et al. IP-10response to RD1 antigens might be a useful biomarker for monitoring tuberculosis therapy. BMC Infect Dis, 2011, 11:135. 